\title{
Exploring variability in environmental flow metrics for assessing options for farm dam low flow releases
}

\author{
$\underline{\text { L.T. Cetin }}^{\text {a }}$, M.R. Alcorn ${ }^{\text {b }}$, J. Rahmanc and K. Savadamuthu ${ }^{\text {b }}$ \\ ${ }^{a}$ Sinclair Knight Merz, Victoria, ${ }^{b}$ Department of Environment, Water and Natural Resources, Adelaide, South \\ Australia, ${ }^{c}$ Flow Matters Pty Ltd, Australian Capital Territory. \\ Email:1cetin@globalskm.com
}

\begin{abstract}
The South Australian Department of Environment, Water and Natural Resources (DEWNR) has undertaken a study to assess the strategic placement of low flow releases (LFR) on farm dams in three Water Resources Areas of the Mount Lofty Ranges, with the purpose of maintaining healthy water dependent ecosystems whilst balancing consumptive demands. Hydrological modelling was undertaken using eWater Source of different LFR installation options based on policies outlined in the draft Water Allocation Plans for the region and optimizing the number and location of LFRs. To facilitate evidence-based decision making as to the optimal set of LFR scenarios that meet environmental flow targets an uncertainty and parameter sensitivity analysis was undertaken.
\end{abstract}

The aims of the uncertainty analysis were to:

1. Determine the optimal scenario of low-flow release installation options based on the outcomes of the uncertainty and parameter sensitivity analysis.

2. Understand the sensitivity of key farm dam model parameters on the outcomes of each scenario.

3. Determine if certain environmental flow metrics are more sensitive to changes in flow than others.

Hydrological modelling using eWater Source and the Farm Dam Analysis Tool plugin was undertaken to investigate the effects of various LFR placement configurations within selected surface water management zones. Through stakeholder engagement, a range of scenarios was produced to assess the optimal number of LFRs required and the environmental benefit likely to be gained by these options. The relative success of each model scenario was assessed against the environmental water requirements (EWR) for the study regions through the use of environmental flow metrics (EFM), which rely on meeting EWRs related to flow regime, on-farm water usage, dam volumes and low flow release rates.

The EFM framework involves the calculation of 58 different flow metrics, with different pass/fail criteria. As a result of the complexity inherent in the EFM framework, the importance and correlation between environmental flow metrics was investigated using principle component analysis (PCA). A parameter sensitivity analysis using Monte-Carlo methods was conducted on the water use fraction parameter, evaporation loss and dam volume relationship of the farm dam model as these parameters have been identified by previous studies as having a high impact on the uncertainty on model outputs.

From the PCA, five metrics were strongly indicative in differentiating between scenario outcomes. These five metrics generally relate to the occurrence and duration of freshes and high seasonal flows, which are strongly affected by the impoundment of runoff by farm dams during dry periods. The Monte Carlo analysis found that the overall variability in metric pass/fail score for each farm dam parameter was not large, with standard deviations generally between $0.004-0.05$. The evaporation loss parameter accounted for the largest source of model uncertainty, which is consistent with the literature. Those metrics that exhibited the highest degrees of variability were consistent with the outcomes of the PCA.

The sensitivity in farm dam parameters on scenario outcomes had little impact on the overall selection of the preferred LFR option. However, the variability attributed to certain pass/fail criteria of individual metrics did aid in differentiating between options that produced similar levels of water recovered and performance in meeting environmental flow criteria. The end results of the preferred LFR options are reinforced by the uncertainty analysis, and give support to the project recommendations that will aid in policy decision making.

Keywords: Uncertainty analysis, Parameter sensitivity, Low flow releases, Environmental water requirements, eWater Source, farm dam modelling 
Cetin et al., Exploring variability in environmental flow metrics for assessing options for farm dam low flow releases

\section{INTRODUCTION}

The South Australian Department of Environment, Water and Natural Resources (DEWNR) has undertaken a feasibility study to assess the strategic placement of low flow releases (LFR) on farm dams in three Prescribed Water Resources Areas of the Mount Lofty Ranges, with the purpose of maintaining healthy water dependent ecosystems whilst balancing consumptive demands (Alcorn et al, 2012). Low flow bypass installations prevent low flows from being captured by farm dams for the purpose of maintain minimum environmental streamflow requirements, particularly during dry periods.

The three prescribed areas are (Figure 1):

1. Marne Saunders Prescribed Water Resources Area (MS)

2. Eastern Mount Lofty Ranges Prescribed Water Resources Area (EMLR)

3. Western Mount Lofty Ranges Prescribed Water Resources Area (WMLR)

All three regions have surface and groundwater resources that are prescribed (or allocated) to particular water users, with a requirement for drafts Water Allocation Plans (WAP) that outlines provisions for the competing uses of the environment and other consumptive requirements. The Marne-Saunders draft Water Allocation Plan has been in place since 2010, while the draft EMLR and WMLR Plans are currently being finalised. The key policy drivers for LFRs in all three draft WAPs are a requirement to release low flows that are below a site-specific threshold flow rate, from existing and new farm dams and maintain threshold flow rates for existing and new diversions from streams. This is expected to "provide sufficient flow and a flow regime that provides an acceptable level of risk to the water-dependent ecosystems" (SAMDB NRM Board, 2011). All three draft WAPs, through a detailed assessment of Environmental Water Requirements (EWRs), identified the critical need for low flows to pass through the system and that this part of the flow regime is currently the most impacted, particularly in the early flow seasons from around May-July and also during the summer low flow season (Vanlaarhoven and Van Der Wielen, 2009).

The current estimate of dams that would require the installation of some infrastructure to release low flows over the three prescribed areas is approximately 2,500. Whilst extensive modelling of varying water usage rates from dams was explored during the development of the draft WAPs, only a single scenario was investigated for placement of low flow releases. As a result, new hydrological modelling was undertaken within this project of different LFR installation options based on policies outlined in the draft WAPs for the region and aims to minimise the number, and optimise the location, of LFRs. To facilitate evidence-based decision making as to the optimal set of LFR scenarios that meet environmental flow targets an uncertainty and parameter sensitivity analysis was undertaken and this is the focus of this paper.

Uncertainty considerations will determine whether a model is fit for purpose and can often aid in exploring solution options, as uncertainties may alter choices or initiate new investigations. Black et al (2011) give a comprehensive overview of uncertainty analysis and options modelling as well as questions or strategies that the modeller should consider when performing scenario modelling.

The aims of the uncertainty and parameter sensitivity analysis were to:

1. Determine the optimal scenario based on the outcomes of the uncertainty and parameter sensitivity analysis.

2. Understand the sensitivity of key farm dam model parameters on the outcomes of each scenario.

3. Determine if certain environmental flow metrics are more important than others.

\section{METHODOLOGY}

The methodology utilised for this project included modelling the effects of various placement configurations of LFRs within selected surface water management zones and identifying different volumes of water that could be returned to the system as a result of the implementation of any particular scenario. An initial stage of visual and numerical data exploration yielded a set of placement scenarios. It was not intended, within the current project timelines, to undertake on-site or localised data assessments, or consider social and economic factors.

There are a total of 584 surface water management zones (SWMZ) in the study area (Figure 1) and the level and pattern of farm dam development varies widely across the area. A detailed analysis of the entire region was considered outside the scope of this project, and consequently 10 'test zones' were selected for exploring LFR optimisation scenarios. The 10 zones selected represent a wide range of hydrologic and geographic characteristics of all zones within the region. 
Cetin et al., Exploring variability in environmental flow metrics for assessing options for farm dam low flow releases

Through stakeholder engagement, a range of scenarios were produced (Table 1) to assess the optimal number of LFRs required and the environmental benefit likely to be gained by these options. Scenario outcomes were compared to Scenario 3 as the "Base-WAP" is the current policy arrangement and has the highest number of LFR installations.

\subsection{EWR Testing Criteria}

The relative success of each model scenario was assessed against the EWRs for the EMLR and WMLR regions through the use of Environmental Flow Metrics, which rely on meeting EWRs related to flow regime, on-farm water usage, dam volumes and low flow release rates.

Vanlaarhoven and van der Wielen's (2009) Environmental Flow Metrics framework involves the calculation of 58 different flow metrics, some for annual flow criteria and others across 4 flow seasons. In order to have some flexibility within the framework at an acceptable level of deviation, a site is required to pass at least $85 \%$ of all priority 1 metrics, which are metrics representing critical ecological functions for maintaining ecosystem health. The Base-WAP scenario (scenario 3), with its requirement to release low flows below a site-specific threshold flow rate defined in each WAP, from all licensable dams, is generally very effective at meeting the $85 \%$ target threshold, but not in all zones tested. In those cases, the nominal target for scenarios 4 to 7 is that the percentage of metrics failed does not increase. Responding to the complexity inherent in the EFM framework, the importance and correlation

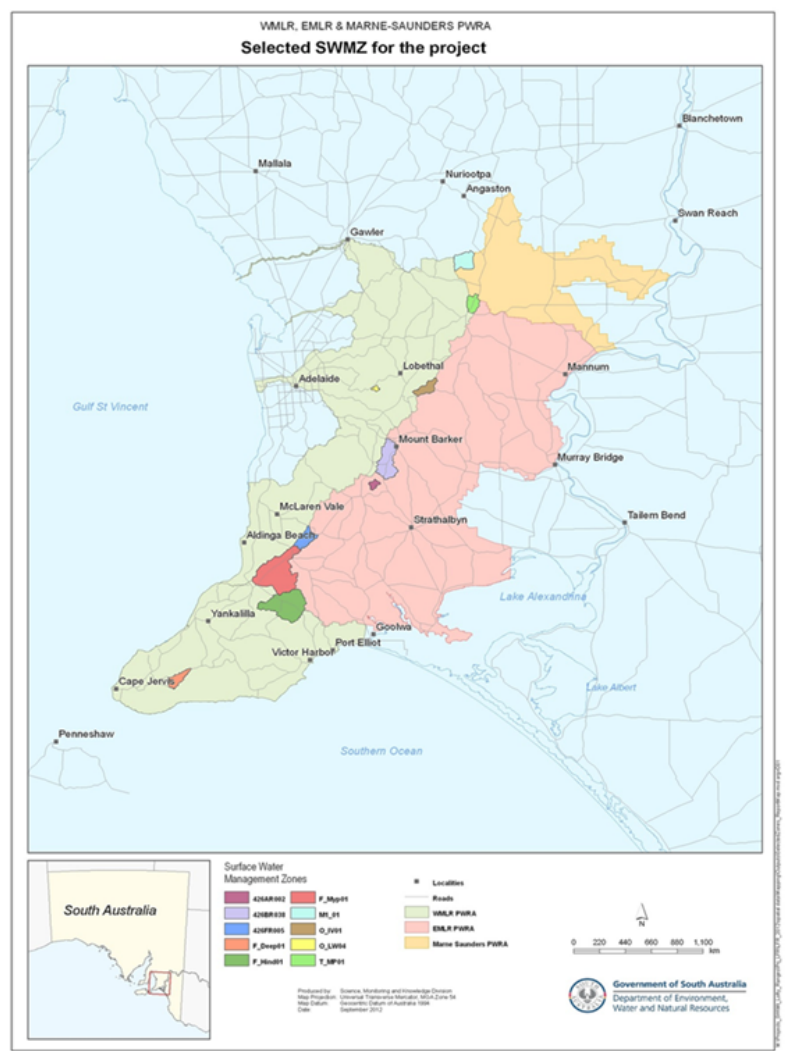

Figure 1. Location Map of the WMLR, EMLR and MS Prescribed Water Resources Areas and the selected test zones for hydrological modelling of low flow bypass installation.

Table 1. Scenario descriptions.

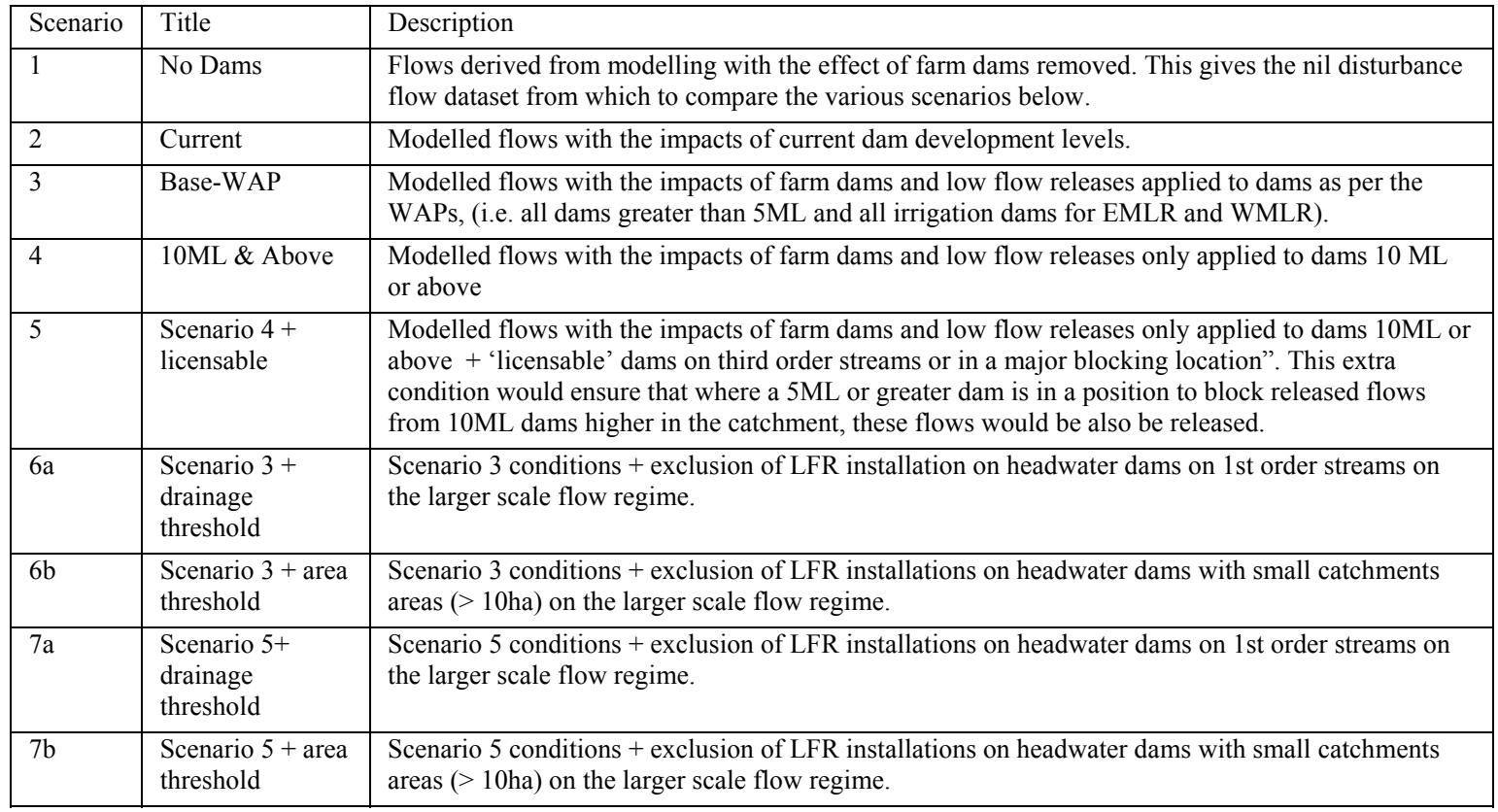


Cetin et al., Exploring variability in environmental flow metrics for assessing options for farm dam low flow releases

between environmental flow metrics was investigated using principle component analysis (PCA).

\subsection{Model development and simulation}

Under the National Hydrological Modelling Strategy, eWater Source (Welsh, et al., 2012) was selected as the modelling platform for water allocation planning (version 3.0.7.31). A river system can be configured as a node-link network where flow is routed through the system and modified by sources and sinks, such as irrigation, urban drinking water supply, and reservoir management. Upstream catchments can be described spatially to enable processes such as rainfall-runoff to deliver flow to the river network. In order to explicitly model the spatial representation of farm dams within a catchment, a customised plugin, the Farm Dam Analysis Tool, developed jointly between Sinclair Knight Merz (SKM) and Western Australia's Department of Water, was used (Fowler, et al., 2012). The plugin provides all the requirements to model the spatial representation of farm dams configured with low flow bypass installations and the effects these dams will have on end of catchment flows.

A Source model for each test zone was constructed to simulate the catchment hydrology and explicit location and characteristics of each dam (see Alcorn et al, 2012 for model build details). Each test zone model was run for the period of 1974-2006 at a daily timestep in accordance with the modelling period of previous EWR analysis for the EMLR.

\subsection{Uncertainty and parameter sensitivity analysis}

Variability between environmental flow metrics

As there are a total of 58 individual environmental flow metrics, it was important to understand and identify which metrics were the most sensitive to changes between scenarios. Therefore, a principle component analysis (PCA) was performed to differentiate between the most critical metrics. Although various flow metrics describe different aspects of the flow regime, most of them are strongly intercorrelated. PCA is a mathematical method that transforms a matrix of possibly correlated variables into a set of linearly uncorrelated variables, or principle components. Each principle component is a measure of the variability within the matrix, and is a way of identifying patterns in data. The PCA was performed using the R statistical software (specifically the prcomp() function). Table 2 outlines the metrics analysed by PCA after discarding those metrics that passed under all scenarios and those with pass/fail scores (expressed as a percentage) that did not change substantially $( \pm 10 \%)$ between each scenario when compared to scenario 2 (current level of farm dam development).

Table 2. List and description of the environmental flow metrics that were analysed in the PCA, after filtering out those that did not change substantially between scenarios and that all passed the pass/fail criteria. *Low flow freshes refer to relatively small, short duration high flow events that last for up to several days. ** High flow freshes are long, sustained increases in flow that may last for several weeks but are contained within the channel

\begin{tabular}{|l|l|l|l|}
\hline ID & Flow Season & Flow Event & Statistic \\
\hline A & Annual & Bankfull & Number of years with one or more flow component \\
\hline B & Low flow & Low flow & 80 percentile exceedance non-zero flow \\
\hline C & Low flow & *Freshes & Number of years with one or more flow component \\
\hline D & Low flow & *Freshes & Average number of flow component per year \\
\hline E & Low flow & *Freshes & Average total duration of flow component per year \\
\hline F & T1 - Transitional (Low-High) & Low flow & 80 percentile exceedance non-zero flow \\
\hline G & T1 - Transitional (Low-High) & Low flow & Current month reaching median flow of natural T1 median (delay) \\
\hline H & T1 - Transitional (Low-High) & **Freshes & Average number of flow component per year \\
\hline I & T1 - Transitional (Low-High) & **Freshes & Average total duration of flow component per year \\
\hline J & T1 - Transitional (Low-High) & **Freshes & 80 percentile exceedance non-zero flow \\
\hline K & T1 - Transitional (Low-High) & High flow & Average total duration of flow component per year \\
\hline L & T2 - Transitional (High-Low) & Low flow & Median non-zero daily T2 flow \\
\hline M & T2 - Transitional (High-Low) & Low flow & 80 percentile exceedance non-zero flow \\
\hline N & T2 - Transitional (High-Low) & Low flow & Current month reaching median flow of natural T2 median (early onset) \\
\hline O & T2 - Transitional (High-Low) & **Freshes & Number of years with one or more flow component \\
\hline P & T2 - Transitional (High-Low) & **Freshes & Average number of flow component per year \\
\hline Q & T2 - Transitional (High-Low) & **Freshes & Average total duration of flow component per year \\
\hline
\end{tabular}


Cetin et al., Exploring variability in environmental flow metrics for assessing options for farm dam low flow releases

\section{Parameter sensitivity analysis}

Understanding which parameters cause a large degree of change in the outcomes of the LFR scenarios can give insight into where areas of uncertainty lie within the model structure or data used to parameterise the model. In an analysis of the TEDI farm dam model parameters, Lowe and Nathan (2008) demonstrated that the volume regression relationship parameters caused the volume ofof farm dams in the Werribee catchment (Vic) varied by $\pm 11 \%$ of the mean and the overall catchment scale impacts on runoff varied by $\pm 29 \%$ of the mean. Fowler et al (2012) found that uncertainties in the spatial variation of flow generation as an input to the Source Farm Dam Analysis Tool plugin were the greatest source of uncertainty in model outputs. In a South Australian context, the studies conducted by McMurray $(2003 ; 2004)$ on dams in the Mount Lofty ranges indicate that potential sources of error in farm dam parameters and relationships are the digitising errors that occurred in defining the dam water outline from aerial photography, farm dam water use fraction, evaporation loss and the errors associated with the farm dam volume-surface area regression relationship. As a result, the water use fraction parameter, evaporation loss and dam volume relationship was subjected to an uncertainty analysis using a Monte Carlo approach.

Normal distributions for each of the three farm dam parameters were sourced from the literature based on previous studies by McMurray (2004) on uncertainties in farm dam model parameterization:

- Dam Volume: dam volumes are derived from a regression relationship to surface area. A scaling factor was used to linearly scale dam volumes for each iteration of Monte Carlo runs. The scaling factor was given a normal distribution with a mean of 1 and standard deviation of 0.25 .

- Evaporation loss: The rate of evaporation from the farm dams' surface area (itself related to surface area volume relationship at less than full capacity) was modified with a linear scaling factor that was applied to the evaporation time series for each Monte Carlo iteration. The linear scaling factor was given a normal distribution with a mean of 1 and standard deviation of 0.2 .

- Dam usage fraction: Assumptions of the demand from farm dams is based on dam size and purpose. It was assumed that demand from large or irrigation dams is a larger proportion (50\%) than smaller dams used for stock and domestic purposes. For each Monte Carlo run, the dam usage fraction was modified based on a normal distribution with a mean of 0.46 (ie, $46 \%$ of dam volume) and standard deviation of 0.25 . This is consistent with the distribution for dam use given by Fowler et al (2012).

The Monte-Carlo simulations were implemented in R using a new package, SourceRy, with global variables that varied each of the farm dam parameters within its normal distribution. SourceRy simplifies the task of working with Source models from $\mathrm{R}$ and includes support for managing multiple scenarios, monte-carlo runs and for running multiple Source models in parallel on multi-core computers. Following this project, SourceRy has been made available from http://github.com/flowmatters/sourceRy. Initially, 1000 Monte Carlo runs of each farm dam parameter were undertaken, but due to computational issues this was reduced to 300 runs for each farm dam parameter. Although this is generally a very low number of runs to generate sufficient replicates to capture the sensitivity range of each parameter, comparison of the 1000 runs and 300 runs for evaporation loss shows that the effect on the results is minimal (Figure 2).

\section{RESULTS AND DISCUSSION}

\subsection{Differentiation in scenario outcomes based on key environmental flow metrics}

The PCA identified eight principle components, with $91 \%$ of the variance in the data matrix attributed to the first principle component and the second principle component accounting for a further $7 \%$ of the variability (up to 98\%). A biplot of the first two principle components shows the groupings of correlated scenarios and environmental flow metrics (Figure 4). Each object's (scenario) and each variable's (metric) distance from the origin $(0,0$ coordinate) is the deviation from its mean; thus a large distance from the origin means a higher variance from the mean. Four distinct groupings of scenarios can be seen in the biplot (blue squares in Figure 3). Scenario 2 and scenario 4 are not correlated with each other or any of the other scenarios, and their distance from the origin is the largest, suggesting that these two scenarios contribute to a high degree of variability in the overall data matrix. Scenarios $6 a$ and $7 \mathrm{a}$ are similar to each other, which is not surprising as 
Cetin et al., Exploring variability in environmental flow metrics for assessing options for farm dam low flow releases

both these scenarios investigate the exception of installing low flow bypasses on dams on first order streams. Scenarios 3, 5, 6b and $7 \mathrm{~b}$ form another correlated group, and are the closest grouping to the origin coordinate, suggesting the lowest variance between variables. As all LFR installation scenarios will be assessed against scenario 3 outputs, it can be concluded from the groupings in the PCA that scenarios 5, 6b and $7 \mathrm{~b}$ are similar to scenario 3 and will effectively produce comparable outcomes with less low flow bypass installations. The key measures of the degree of correlation between environmental flow metrics are the length of the vectors (the red arrows), which shows the degree of variance associated with an individual metric, and the angle between vectors, which illustrates the degree of correlation (Figure 3). Five grouping of environmental flow metrics can be delineated from the biplot in Figure 3. The main pattern emerging between each correlation group is the grouping of low flow season/"high to low transitional" flows season metrics (groups 2, 3 and 4), the"low to high transitional" flow season metrics (group 5) and the bankfull/high flow season metrics (group 1).

The metrics that account for the greatest degree of variability are:

- Metric A - number of years with one or more bankfull flows

- Metric B - low Flow Season - 80 percentile exceedance non-zero flows

- Metric L - median non-zero daily T2 low flow

- Metric D - average number of low flow season freshes per year

- Metric E - Average total duration of low flow season freshes per year

\subsection{Environmental flow metric sensitivity Monte Carlo analysis}

Although the parameter sensitivity analysis was conducted for all scenarios, only results for scenario 3 are presented here, as scenario 3 has the most LFRs and represents the best case of recovering the greatest volume of water. Overall, the variability in metric pass/fail score for each farm dam parameter was not large, with

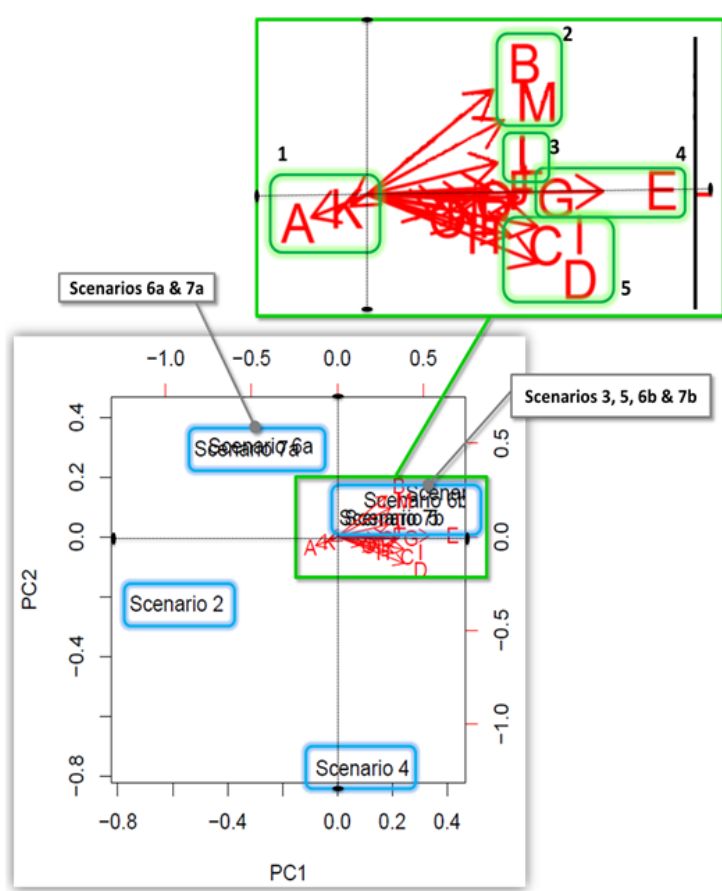

Figure 3. PCA biplot distinguishing between scenarios (blue groups) and environmental flow metric pass/fail score variables (green groups).

Dotted intersecting lines show the 0,0 origin.

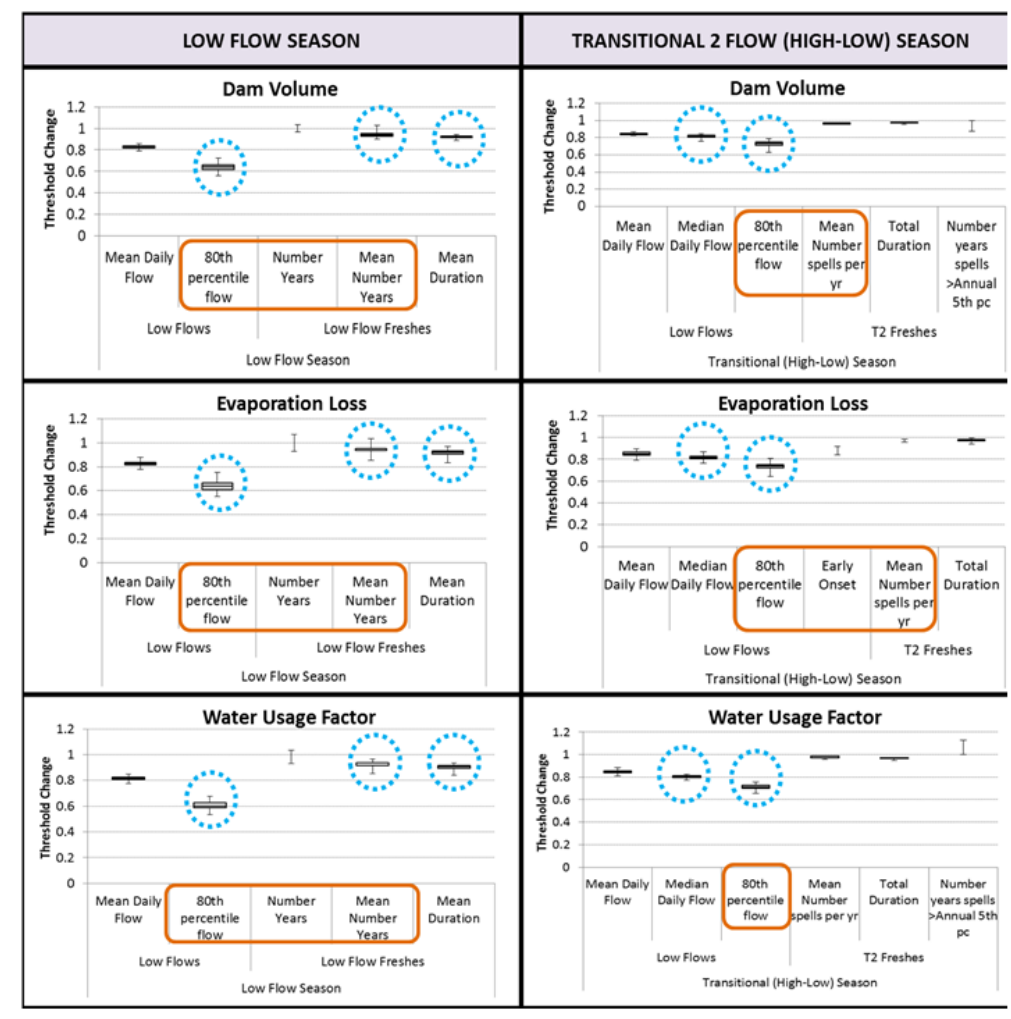

Figure 4. Sensitivity in pass/fail score for low flow and "high to low transitional" flow season metrics for each farm dam parameter. Orange boxes denote priority 1 metrics that require a pass score of $85 \%$. Blue circles denote metrics identified in the PCA as the most variable. Those metrics which showed no distinct variability have 
Cetin et al., Exploring variability in environmental flow metrics for assessing options for farm dam low flow releases

standard deviations generally between $0.004-0.05$. Across all environmental flow metrics, the evaporation loss parameter was the most sensitive, with the usage fraction parameter producing the least sensitive response (Figure 4). This is consistent with the findings from McMurray (2004). Those metrics that showed the most sensitivity to each of the farm dam parameters tested (blue circles in Figure 4) were predominantly metrics associated with the Low Flow Season and "high to low transitional" flow season, particularly the 80th percentile non-zero flows and the mean number of years with 1 or more freshes, and is consistent with the findings from the PCA. With respect to priority 1 metrics (indicated by orange squares in Figure 4), there are no instances where a metric may switch from pass to fail, or vice versa. This suggests that although there is variability in the metric threshold change score caused by the farm dam parameters, the variability is not of sufficient magnitude to influence the overall end results of the scenario analysis.

\section{CONCLUSIONS}

A sensitivity analysis was undertaken to explore the uncertainty within the farm dam model, the interrelationships between environmental flow metrics and how this may influence the strategic placement of low flow release installation on farm dams. The sensitivity in farm dam parameters on scenario outcomes had little impact on the overall selection of the preferred LFR option. However, the variability attributed to certain pass/fail criteria of individual metrics did aid in differentiating between options with similar outcomes. The end results of the preferred LFR options are reinforced by the uncertainty analysis, and give support to the project recommendations that will aid in policy decision making.

This investigation to explore the inter-relationships between environmental flow metrics is preliminary, and a more in-depth methodology could be incorporated into the standard EWR assessment framework to provide a quantitative asset to policy development and implementation.

\section{REFERENCES}

Alcorn M.R., Savadamuthu K, Cetin, L, Shrestha P, (2012), Strategic \& prioritised approach to location of Low Flow Releases in The Mount Lofty Ranges - Feasibility Study, Department of Environment, Water and Natural Resources, South Australia.

Black, D., Wallbrink, P., Jordan, P., Waters, D., Carroll, C., \& Blackmore, J. (2011). eWater Cooperative Research Centre, Guidelines for water management modelling: Towards best-practice model application, Canberra.

Fowler, K., Donohue, R., Morden, R., Durrant, J., Hall, J., Narsey, S. (2012). Decision support using Source IMS for licensing and planning in self supply irrigation areas. River Symposium, Melbourne.

Fowler, K., Morden, R., Wiesenfeld, C., Delaney, P., Kiely, N., Walpole, L. (2011). Updating regional farm dam characteristics for use across the Murray Darling Basin. Proceedings of the 34th World Congress of the International Association for Hydro-Environment Engineering and Research (IAHR), Brisbane.

Lowe, L., \& Nathan, R. (2008). Consideration of uncertainty in the estimation of farm dam impacts. Proceedings of the Water Down Under conference, Adelaide.

McMurray, D. (2003). Assessment of Water Use from Farm Dams in the Mount Lofty Ranges South Australia. Department of Water, Land and Biodiversity Conservation, South Australia.

McMurray, D. (2004). Farm Dam Volume Estimations from Simple Geometric Relationships. Department of Water, Land and Biodiversity Conservation, South Australia.

SAMDB NRM Board. (2011). Draft Water Allocation for the Eastern Mount Lofty Ranges Prescribed Water Resources Area. South Australian Murray-Darling Basin Natural Resources Management Board, Government of South Australia.

VanLaarhoven, J. M., \& Van der Wielen, M. (2009). Environmental Water Requirements for the Mount Lofty Ranges prescribed water resources area. Department of Water, Land and Biodiversity Conservation \& South Australian Murray-Darling Basin NRM Board.

VanLaarhoven, J., \& Van der Wielen, M. (2012). Assessment of the needs of water dependent ecosystems for the Eastern Mount Lofty Ranges Prescribed Water Resources Area. Department for Water, Government of South Australia.

Welsh WD, Vaze J, Dutta D, Rassam D, Rahman JM, Jolly ID, Wallbrink P, Podger GM, Bethune M, Hardy M, Teng J, Lerat J. (2012). An integrated modelling framework for regulated river systems. Environmental Modelling and Software, 39, 81-102. 